\title{
Uso de eugenol como anestésico em pacu ${ }^{1}$
}

\author{
Daniel Antonio Rotili², Maicon Adelio Devens², Odair Diemer ${ }^{3}$, \\ Evandro Kleber Lorenz ${ }^{3}$, Rafael Lazzari ${ }^{2}$, Wilson Rogério Boscolo ${ }^{3}$
}

\section{ABSTRACT}

\section{Eugenol as anesthetic for Piaractus mesopotamicus}

The different practices used in fish breeding require intense handling, leading to fish stress. The use of anesthetics is important to overcome this problem. This study aimed at evaluating the impact of eugenol on the induction and anesthetic recovery of Piaractus mesopotamicus from different weight classes. An experiment was carried out in a $5 \times 5$ factorial scheme, consisting of five weight classes $(50 \pm 5.61 \mathrm{~g}, 100 \pm 9.36 \mathrm{~g}, 150 \pm$ $12.29 \mathrm{~g}, 200 \pm 12.73 \mathrm{~g}$ and $250 \pm 18.77 \mathrm{~g})$ and five eugenol concentrations ( $40 \mathrm{mg} \mathrm{L}^{-1}, 80 \mathrm{mg} \mathrm{L}^{-1}, 120 \mathrm{mg} \mathrm{L}^{-1}, 160 \mathrm{mg} \mathrm{L}^{-1}$ and $\left.200 \mathrm{mg} \mathrm{L}^{-1}\right)$. The anesthesia induction was divided into four stages, according to the behavior of the anesthetized fish, being the duration of each stage recorded, and the desensitization moment and recovery time evaluated. A quadratic effect was observed at the anesthetic induction time, i.e., when the dose increased, the induction time decreased, tending to stabilize for increasing doses. The best results for anesthetic induction were observed at the $175 \mathrm{mg} \mathrm{L}^{-1}, 208 \mathrm{mg} \mathrm{L}^{-1}, 203 \mathrm{mg} \mathrm{L}^{-1}, 240 \mathrm{mg} \mathrm{L}^{-1}$ and $225 \mathrm{mg} \mathrm{L}^{-1}$ concentrations, respectively for the $50 \mathrm{~g}, 100 \mathrm{~g}$, $150 \mathrm{~g}, 200 \mathrm{~g}$ and $250 \mathrm{~g}$ weight classes. No interaction between the shortest induction time and the shortest recovery time was observed. Thus, the doses which provided a lower induction time for the weight classes range between $175 \mathrm{mg} \mathrm{L}^{-1}$ and $240 \mathrm{mg} \mathrm{L}^{-1}$.

KEY-WORDS: Piaractus mesopotamicus (Holmberg); aquaculture; fish management.

\section{INTRODUÇÃO}

O pacu (Piaractus mesopotamicus, Holmberg, 1887) é um peixe nativo das bacias dos rios Paraná, Uruguai e Paraguai e adapta-se facilmente aos sistemas de cultivo intensivo. Devido ao alto valor comercial da sua carne, aceitação da alimentação artificial e rápido crescimento, é largamente utilizado na piscicultura brasileira (Bittencourt 2008).

\section{RESUMO}

Os diferentes manejos utilizados na piscicultura demandam intenso manuseio, causando estresse aos peixes. $\mathrm{O}$ uso de anestésicos é importante para contornar este problema. Este trabalho foi realizado para avaliar o uso de eugenol na indução e recuperação anestésica, em pacus de diferentes classes de peso. Foi desenvolvido um experimento em esquema fatorial $5 \times 5$, composto de cinco classes de peso $(50 \pm 5,61 \mathrm{~g} ; 100 \pm 9,36 \mathrm{~g} ; 150 \pm 12,29 \mathrm{~g}$; $200 \pm 12,73 \mathrm{~g}$; e $250 \pm 18,77 \mathrm{~g}$ ) e cinco concentrações de eugenol (40 mg L-1, $80 \mathrm{mg} \mathrm{L}^{-1}, 120 \mathrm{mg} \mathrm{L}^{-1}, 160 \mathrm{mg} \mathrm{L}^{-1}$ e $200 \mathrm{mg} \mathrm{L}^{-1}$ ). A indução da anestesia foi dividida em quatro estágios, de acordo com o comportamento dos peixes sob efeito do anestésico, registrando-se o tempo de permanência em cada estágio, sendo avaliado o momento de insensibilização e o tempo de recuperação. Para o tempo de indução anestésica, observou-se efeito quadrático, ou seja, quando aumentou-se a dose, houve queda no tempo de indução, com tendência de estabilização em doses crescentes. As melhores respostas de indução anestésica verificadas para o pacu ocorreram nas dosagens de $175 \mathrm{mg} \mathrm{L}^{-1}, 208 \mathrm{mg} \mathrm{L}^{-1}, 203 \mathrm{mg} \mathrm{L}^{-1}$, $240 \mathrm{mg} \mathrm{L}^{-1}$ e $225 \mathrm{mg} \mathrm{L}^{-1}$, respectivamente para as classes de peso de 50 g, 100 g, 150 g, 200 g e 250 g. Já para o tempo de recuperação, não foi observada interação entre o menor tempo de indução e menor tempo de recuperação. Sendo assim, as doses que proporcionaram menor tempo de indução para as faixas de peso encontram-se entre $175 \mathrm{mg} \mathrm{L}^{-1}$ e $240 \mathrm{mg} \mathrm{L}^{-1}$.

PALAVRAS-CHAVE: Piaractus mesopotamicus (Holmberg); aquicultura; manejo de peixe.

Os manejos como biometria, análise patológica, implantes hormonais e transporte podem causar ferimentos e perdas de escamas, além de expor os peixes a uma variedade de fatores estressantes, que podem afetar seu desempenho produtivo e até levá-los à morte (Barton 2000). As consequências dos danos causados aos peixes incluem o aumento da susceptibilidade a doenças patogênicas e infecciosas (Yukihiro et al. 2008). Além disto, movimentos

1. Trabalho recebido em mar./2012 e aceito para publicação em ago./2012 ( ${ }^{\circ}$ registro: PAT 17688).

2. Universidade Federal de Santa Maria (UFSM), Departamento de Zootecnia e Ciências Biológicas, Palmeira das Missões, RS,

Brasil.E-mails: daniel_rotili@hotmail.com,devens1980@hotmail.com,rlazzari@ufsm.br.

3. Universidade Estadual do Oeste do Paraná (Unioeste), Centro de Engenharias e Ciências Exatas, Departamento de Engenharia de Pesca, Toledo, PR, Brasil.E-mails: odairdiemer@hotmail.com, evandrolorentz@hotmail.com, wilsonboscolo@hotmail.com. 
bruscos dos peixes colocam em risco, também, a segurança dos trabalhadores (Barbosa et al. 2007).

O uso de anestésicos demonstra eficácia no controle de eventuais desconfortos e facilita o manejo geral, além de proporcionar menor risco ao manipulador. A escolha de um anestésico deve estar relacionada com sua viabilidade econômica, praticidade no uso, eficácia e considerações legais (Cho \& Heath 2000), assim como ação rápida sobre o sistema nervoso, sem complicações posteriores para o peixe (Gonçalves et al. 2008).

As substâncias anestésicas mais utilizadas são tricaína metano sulfonato (MS-222), quinaldina e 2-fenoxietanol, com custos significativos e efeitos adversos, como irritabilidade e efeitos deletérios nos manuseadores (Roubach \& Gomes 2001). A benzocaína é um dos anestésicos mais utilizados no Brasil, em algumas etapas de manejo de peixes (Oliveira et al. 2009). Outro anestésico bastante utilizado é o eugenol, cujos estudos sobre a sua utilização surgiram da necessidade de encontrar novas substâncias eficazes, seguras e de baixo custo (Roubach et al. 2005).

O óleo de cravo destaca-se como anestésico alternativo, por ser um produto natural, efetivo, de baixo custo, seguro e de fácil acesso (Inoue et al. 2003). Este óleo tem como princípio ativo o composto fenólico eugenol (4-alil-2-metoxifenol- $\mathrm{C}_{10} \mathrm{H}_{12} \mathrm{O}_{2}$ ), derivado do caule, flores e folhas das árvores Eugenia caryophyllata e E. aromatica (Griffiths 2000).

Até o momento, não foram encontrados traços tóxicos desse produto em animais aquáticos previamente expostos, além do fato de que outras áreas, como a odontologia, a indústria de alimentos e a fabricação de perfumes, utilizam amplamente o produto para humanos, sem a constatação de riscos, inclusive ambientais (Barbosa et al. 2007). Trata-se de um anestésico seguro, de grande eficácia, ampla margem de segurança para o peixe e ausência de toxicidade para o operador, nas doses utilizadas para peixes (Keene et al. 1998). Além disto, sua eficácia é maior que a de outras substâncias já utilizadas (Wagner et al. 2003).

A anestesia em peixes pode ser afetada por fatores biológicos, tais como as diferenças entre as espécies (formato do corpo e tamanho da área branquial) e intraespécies, que são as diferenças de tamanho, variações na taxa metabólica e quantidade de gordura corporal (Roubach \& Gomes 2001).

Alguns estudos já demonstraram a eficácia do óleo de cravo como anestésico para o matrinxã, Brycon cephalus (Inoue et al. 2003), tambaqui,
Colossoma macropomum (Roubach et al. 2005), e juvenis de tilápia, Oreochromis niloticus (Delbon \& Paiva 2012).

Este trabalho objetivou avaliar o tempo de indução e recuperação anestésica, em diferentes classes de peso de pacu, submetidas a diferentes doses de eugenol.

\section{MATERIAL E MÉTODOS}

O estudo foi realizado em fevereiro de 2010, no Centro de Desenvolvimento de Tecnologias para Piscicultura em Tanques-rede, localizado no Refúgio Biológico, no reservatório da barragem da Hidroelétrica de Itaipu, município de Santa Helena, Estado do Paraná (2451'37’S e 54¹9'58'W).

Um total de 200 exemplares de pacu, mantidos em tanque-rede e sob jejum de 24 horas, foram removidos e acondicionados em cinco caixas d'água (uma para cada classe de peso), com a mesma condição de água do local de instalação dos tanques-rede.

$\mathrm{O}$ delineamento experimental constituiu-se de esquema fatorial $5 \times 5$, incluindo cinco classes de peso $(50 \pm 5,61 \mathrm{~g} ; 100 \pm 9,36 \mathrm{~g} ; 150 \pm 12,29 \mathrm{~g}$; $200 \pm 12,73 \mathrm{~g}$; e $250 \pm 18,77 \mathrm{~g})$ e cinco tratamentos (40 mg L ${ }^{-1}, 80 \mathrm{mg} \mathrm{L}^{-1}, 120 \mathrm{mg} \mathrm{L}^{-1}, 160 \mathrm{mg} \mathrm{L}^{-1} \mathrm{e}$ $200 \mathrm{mg} \mathrm{L}^{-1}$ ), correspondentes a concentrações do anestésico eugenol. Este foi diluído em álcool etílico $\left(92,8^{\circ}\right)$, resultando em solução estoque na proporção de $100 \mathrm{mg} \mathrm{mL}^{-1}$ (1:10). Oito peixes de cada classe de peso foram expostos, individualmente, às diferentes concentrações do produto, realizando-se a indução à anestesia em um aquário de vidro (capacidade de $30 \mathrm{~L}$ ), contendo $10 \mathrm{~L}$ de água, com as respectivas doses de anestésico e aeração constante.

A recuperação anestésica dos peixes foi realizada individualmente, também em aquários de vidro, contendo $25 \mathrm{~L}$ de água, com aeração constante e sem anestésico. Os peixes foram acondicionados e o tempo de recuperação aferido quando os peixes restabeleceram o equilíbrio corporal, "nadando horizontalmente" (Woody et al. 2002). A água dos aquários (de indução e recuperação) foi renovada ao término de cada concentração testada.

Os tempos necessários para o estabelecimento dos padrões comportamentais avaliados foram monitorados por meio de cronômetro digital, observando-se quatro estágios de anestesia (Tabela 1).

A ausência de reação a qualquer estímulo foi verificada pelo toque na lateral dos peixes, com um 
Tabela 1. Características comportamentais dos peixes, de acordo com os diferentes estágios de anestesia.

\begin{tabular}{cl}
\hline Estágio & \multicolumn{1}{c}{ Característica de comportamento } \\
\hline I & Movimento opercular visivelmente lento ou errático \\
II & Perda parcial de equilíbrio e dificuldade para manter a posição normal de nado, quando parado \\
III & Perda total de equilíbrio e incapacidade de recuperar a posição vertical de nado ("barriga para cima”) \\
IV & Ausência de reação a qualquer estímulo \\
Recuperado & Recuperação da posição normal de nado e da capacidade de nadar \\
\hline
\end{tabular}

Fonte: Woody et al. (2002).

bastão de vidro. Quando atingiram o estágio IV, os peixes foram retirados do aquário de indução, secos com papel tolha e submetidos a biometria, a fim de simular o processo de manejo, sendo, depois, transferidos para o aquário de recuperação. Para a identificação, foram realizados cortes nas nadadeiras dorsal e caudal, conforme o tratamento, facilitando, assim, a sua identificação, em caso da ocorrência de morte.

Após o restabelecimento dos indivíduos no aquário de recuperação, os mesmos foram transferidos para tanques-rede de $6,0 \mathrm{~m}^{3}$ de volume útil, com dimensões de 2,0 m x 2,0 m x 1,5 m de comprimento, largura e profundidade, respectivamente, onde receberam alimentação e ficaram sob observação, durante 96 horas, após a indução anestésica, para o monitoramento da mortalidade (Vidal et al. 2006).

Os dados obtidos foram submetidos à Anova de duas vias. Os valores de tempo de indução anestésica e recuperação, de acordo com o nível de eugenol, em cada classe de peso, foram submetidos a regressão polinomial $(p<0,05)$. As médias entre as classes de peso foram comparadas pelo teste Tukey $(\mathrm{p}<0,05)$.

\section{RESULTADOS E DISCUSSÃO}

Todos os peixes expostos às concentrações de eugenol (40 mg L $\mathrm{m}^{-1}, 80 \mathrm{mg} \mathrm{L}^{-1}, 120 \mathrm{mg} \mathrm{L}^{-1}, 160 \mathrm{mg} \mathrm{L}^{-1}$ e $\left.200 \mathrm{mg} \mathrm{L}^{-1}\right)$ atingiram, satisfatoriamente, o estágio IV da indução anestésica, caracterizado por anestesia profunda, não respondendo a nenhum estímulo. $\mathrm{O}$ índice de sobrevivência foi de $100 \%$, após o período de 96 horas de indução anestésica, indicando ausência de efeito deletério e tóxico para os peixes.

Em relação ao tempo de indução (Figura 1), observou-se comportamento quadrático em todas as classes de peso testadas $(p<0,05)$. Maior tempo de indução (106 s) foi observado na menor dose testada (40 $\mathrm{mg} \mathrm{L}^{-1}$ ), para os peixes da classe de $50 \mathrm{~g}$, sendo que o menor tempo (13 s) foi observado para os pei- xes de $100 \mathrm{~g}$, na dose de $200 \mathrm{mg} \mathrm{L}^{-1}$, demonstrando que as menores doses de eugenol tendem a aumentar o tempo de indução, fato observado neste experimento e confirmado por Delbon \& Paiva (2012), os quais, ao induzirem exemplares de juvenis de tilápia (Oreochromis niloticus) à anestesia com óleo de cravo, em concentrações de $40 \mathrm{mg} \mathrm{L}^{-1}, 60 \mathrm{mg} \mathrm{L}^{-1}$, $80 \mathrm{mg} \mathrm{L}^{-1}, 100 \mathrm{mg} \mathrm{L}^{-1}$ e $120 \mathrm{mg} \mathrm{L}^{-1}$, relataram que a concentração de $40 \mathrm{mg} \mathrm{L}^{-1}$ correspondeu ao maior tempo de indução $(198,8 \pm 32,91 \mathrm{~s})$.

Quanto à recuperação (Figura 1), as doses que apresentaram o menor tempo foram, respectivamente, $80 \mathrm{mg} \mathrm{L}^{-1}$, para as classes de peso de $50 \mathrm{~g}, 100 \mathrm{~g}$ e $150 \mathrm{~g}$, e $40 \mathrm{mg} \mathrm{L}^{-1}$, para as classes de $200 \mathrm{~g}$ e $250 \mathrm{~g}$. Assim, observou-se que o menor tempo de recuperação não está diretamente relacionado ao menor tempo de indução.

De acordo com Ostrensky et al. (2000) e Gomes et al. (2001), o tempo de recuperação do peixe é influenciado pelo tempo de exposição ao fármaco e pela temperatura. De maneira geral, independentemente da classe de peso e dose utilizadas, os valores mínimo e máximo de recuperação foram, respectivamente, 79 e 271 segundos. Sendo assim, constatou-se que o tempo de recuperação é pouco ou não é influenciado pelas diferentes concentrações, fato também observado por Vidal et al. (2007). Entretanto, o tempo de recuperação não excedeu a 10 minutos, para nenhuma das concentrações, preenchendo os requisitos sugeridos por Ross \& Ross (2008). Para os peixes submetidos à dose de $40 \mathrm{mg} \mathrm{L}^{-1}$, o menor tempo de indução foi observado naqueles com $200 \mathrm{~g}$ (70 s), que, também, apresentaram recuperação mais rápida $(88 \mathrm{~s})$.

Para os peixes submetidos à dose de $80 \mathrm{mg} \mathrm{L}^{-1}$, os menores tempos de recuperação foram observados nas três menores classes de peso $(50 \mathrm{~g}, 100 \mathrm{~g}$ e $150 \mathrm{~g}$ ) (Figura 2). Na dose de $160 \mathrm{mg} \mathrm{L}^{-1}$, o menor tempo de indução foi observado para os peixes da classe de $50 \mathrm{~g}(18 \mathrm{~s})$, enquanto a recuperação foi 
mais lenta nos peixes com $200 \mathrm{~g}(270$ s). Para a maior dose $\left(200 \mathrm{mg} \mathrm{L}^{-1}\right)$, os peixes da faixa de $100 \mathrm{~g}$ apresentaram indução anestésica mais rápida (13 s).

O presente estudo demonstra que, conforme vai-se aumentando a dose do anestésico, observa-se
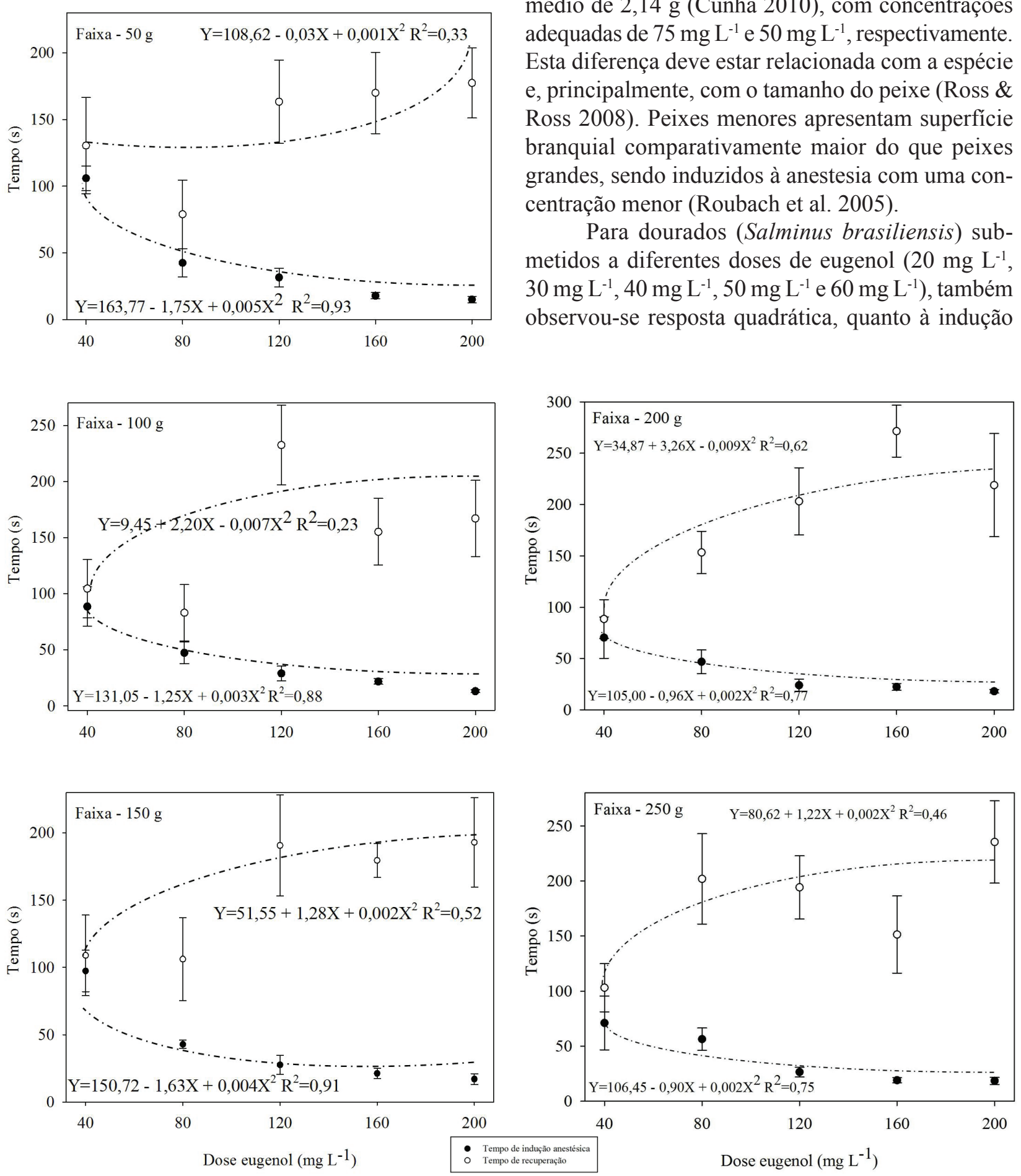

Figura 1. Tempos de indução anestésica e de recuperação de pacus, nas classes de peso de 50 g, 100 g, 150 g, 200 g e 250 g, submetidos a diferentes doses de eugenol (Santa Helena, PR, 2010). uma rápida queda no tempo de indução, o qual tende a ir se estabilizando. Contudo, as concentrações mais eficazes observadas neste estudo, para anestesia profunda, encontram-se acima do intervalo proposto para algumas espécies, como tilápias, com peso médio de 5,34 g (Vidal et al. 2008), e jundiás, com peso médio de 2,14 g (Cunha 2010), com concentrações adequadas de $75 \mathrm{mg} \mathrm{L}^{-1}$ e $50 \mathrm{mg} \mathrm{L}^{-1}$, respectivamente. Esta diferença deve estar relacionada com a espécie e, principalmente, com o tamanho do peixe (Ross \& Ross 2008). Peixes menores apresentam superfície branquial comparativamente maior do que peixes grandes, sendo induzidos à anestesia com uma concentração menor (Roubach et al. 2005).

Para dourados (Salminus brasiliensis) submetidos a diferentes doses de eugenol $\left(20 \mathrm{mg} \mathrm{L}^{-1}\right.$, $30 \mathrm{mg} \mathrm{L}^{-1}, 40 \mathrm{mg} \mathrm{L}^{-1}, 50 \mathrm{mg} \mathrm{L}^{-1}$ e $60 \mathrm{mg} \mathrm{L}^{-1}$ ), também observou-se resposta quadrática, quanto à indução

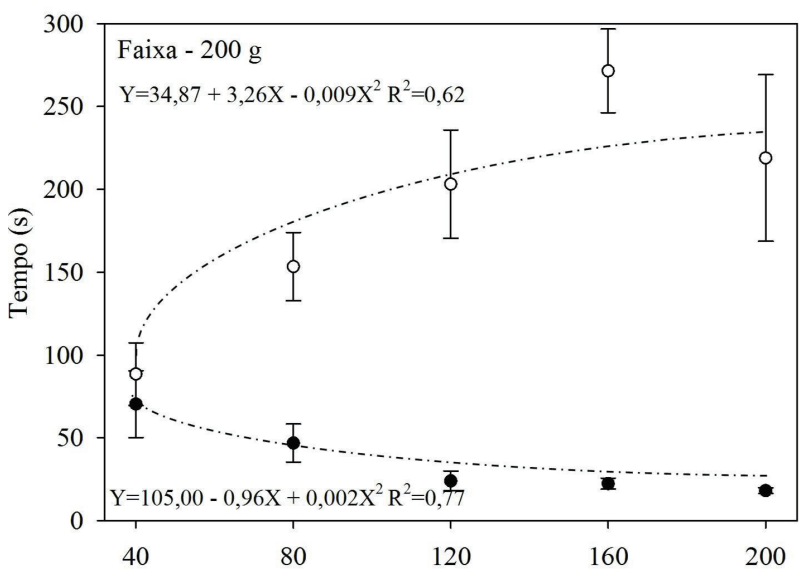


anestésica, sendo a concentração ótima, estimada pela equação $\hat{y}=0,3547 x^{2}-43,307 x+1406,6\left(R^{2}=0,98\right)$, igual a $61 \mathrm{mg} \mathrm{L}^{-1}$ de óleo de cravo (Hisano et al. 2008).

Juvenis de matrinxã (Brycon cephalus) submetidos a diferentes concentrações $\left(18 \mathrm{mg} \mathrm{L}^{-1}\right.$, $20 \mathrm{mg} \mathrm{L}^{-1}, 30 \mathrm{mg} \mathrm{L}^{-1}, 40 \mathrm{mg} \mathrm{L}^{-1}, 50 \mathrm{mg} \mathrm{L}^{-1} \mathrm{e} 60 \mathrm{mg} \mathrm{L}^{-1}$ ) de eugenol não apresentaram diferenças no tempo de indução, para concentrações acima de $40 \mathrm{mg} \mathrm{mL}^{-1}$, sendo o tempo de recuperação independente da concentração do anestésico (Inoue et al. 2003), demonstrando que a concentração de eugenol que
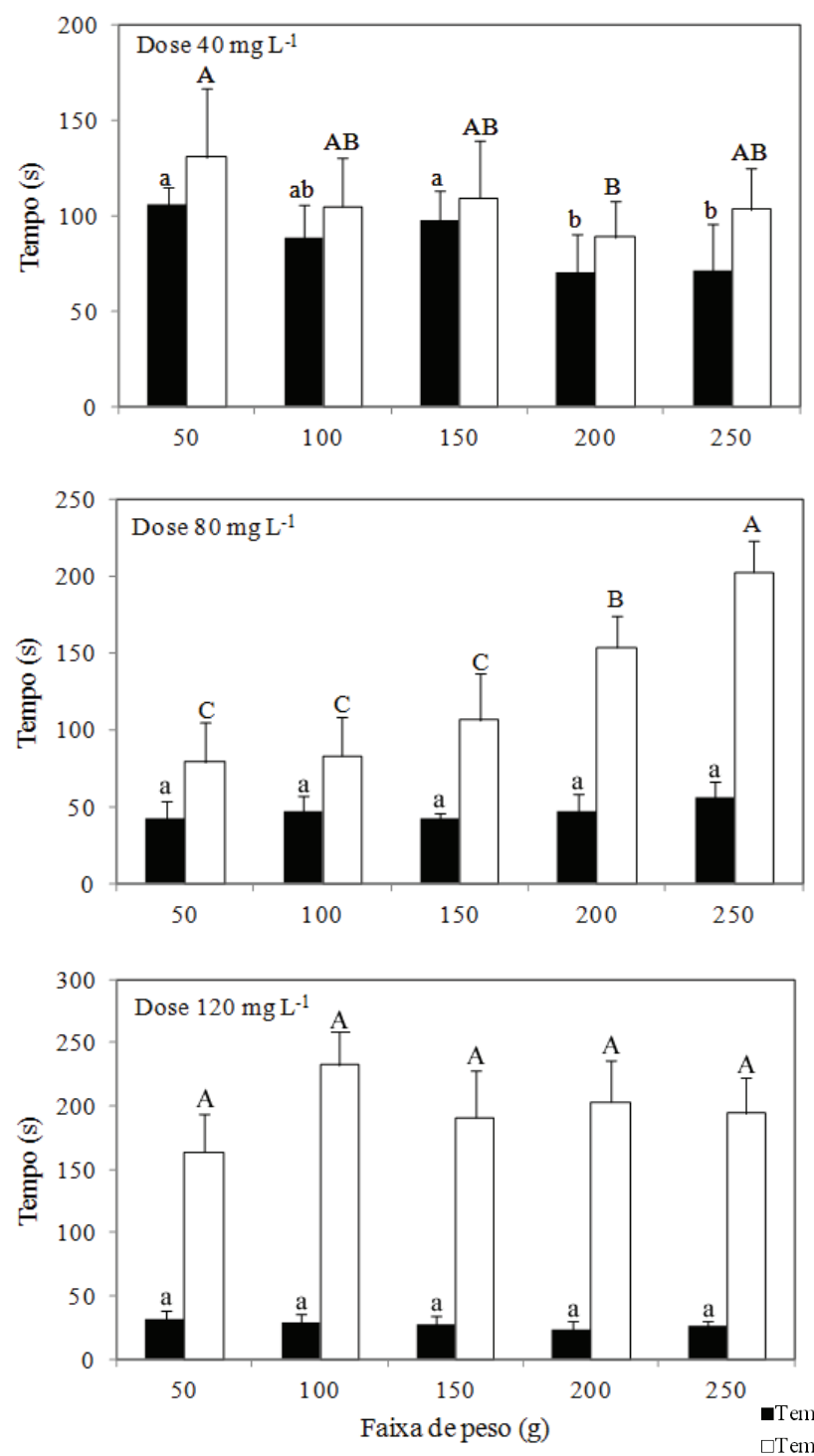

Figura 2. Tempos de indução anestésica e de recuperação de pacus submetidos a diferentes doses de eugenol e classes de peso $(50 \pm 5,61 \mathrm{~g} ; 100 \pm 9,36 \mathrm{~g} ; 150 \pm 12,29 \mathrm{~g} ; 200 \pm 12,73 \mathrm{~g} ;$ e $250 \pm 18,77 \mathrm{~g})$ (Santa Helena, PR, 2010). Letras minúsculas diferentes, nas barras escuras, indicam diferenças significativas $(\mathrm{p}<0,05)$ entre as classes de peso, para tempo de indução anestésica, pelo teste Tukey. Letras maiúsculas diferentes, nas barras claras, indicam diferenças significativas $(p<0,05)$ entre as classes de peso, para tempo de recuperação, pelo teste Tukey.
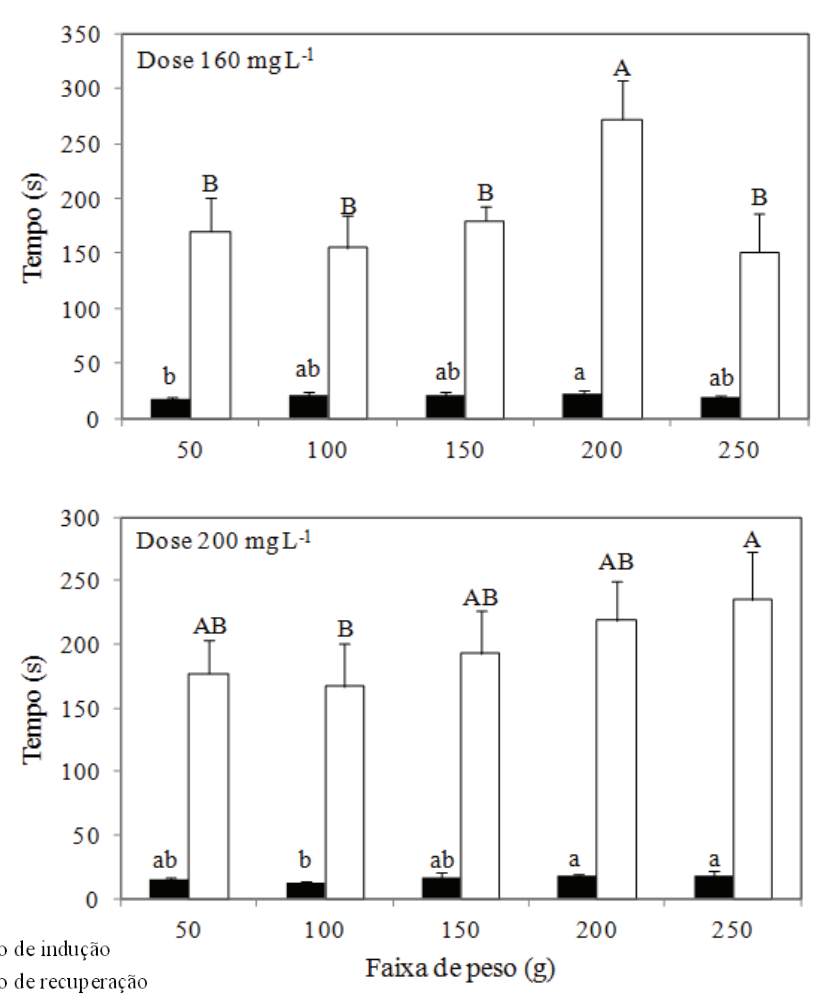

proporciona o menor tempo de indução pode variar conforme a espécie. Entretanto, após determinada dose, o tempo de indução tende a se estabilizar, conforme observado neste estudo.

A utilização de anestésico como ferramenta na piscicultura torna-se cada vez mais indispensável, facilitando, assim, o manejo, pois permite manipulação segura e precisa. O eugenol demonstra eficiência na anestesia de peixes, tornando-se um agente promissor, como anestésico. Entretanto, o efeito fisiológico do eugenol ainda não é claramente conhecido, mas sugere-se que possa interferir no olfato dos peixes. Além concentração de um anestésico pode variar conforme as espécies e o tamanho (Woody et al. 2002).

A utilização de eugenol, em substituição à benzocaína, demonstra que doses de $50 \mathrm{mg} \mathrm{L}^{-1} \mathrm{e}$ $100 \mathrm{mg} \mathrm{L}^{-1}$ de eugenol apresentam tempos de indução iguais aos da dose de $100 \mathrm{mg} \mathrm{L}^{-1}$ de benzocaína, diferindo, apenas, no tempo de recuperação, para a concentração de $100 \mathrm{mg} \mathrm{L}^{-1}$ de eugenol (Gonçalves et al. 2008). disto, para proporcionar o menor tempo de indução, a 
Diferentemente do presente estudo, Vidal et al. (2007), em experimento com matrinxã (3,08-38,45 g) e tambaqui (1,32-33,03 g), utilizando uma dose de $50 \mathrm{mg} \mathrm{L}^{-1}$ de eugenol, concluiram que o peso corporal não apresenta influência sobre o tempo de indução. Estes autores observaram que a anestesia proporcionada pelo eugenol facilitou o manejo de biometria, além de não apresentar efeitos adversos aparentes à saúde dos peixes, fato também constatado por Vidal et al. (2006).

Para o pacu, observou-se que o eugenol pode ser utilizado em todas as classes de peso testadas, não resultando em efeitos adversos aos peixes.

\section{CONCLUSÕES}

1. As doses de eugenol, para pacus com peso variando entre $50 \mathrm{~g}$ e $250 \mathrm{~g}$, que proporcionaram menor tempo de indução encontram-se entre $175 \mathrm{mg} \mathrm{L}^{-1}$ e $240 \mathrm{mg} \mathrm{L}^{-1}$.

2. Em todas as doses testadas, o tempo de recuperação dos peixes foi adequado.

\section{COMITÊ DE ÉTICA E BIOSSEGURANÇA}

Este trabalho foi aprovado pelo Comitê de Ética e Biossegurança da Unioeste (PR), sob o protocolo de número 49/09.

\section{REFERÊNCIAS}

BARBOSA, L. M. G. et al. Respostas metabólicas do matrinxã (Brycon amazonicus) submetido a banhos anestésicos de eugenol. Acta Scientiarum Biological Sciences, Maringá, v. 29, n. 3, p. 255-260, 2007.

BARTON, B. A. Salmonid fishes differ in their cortisol and glucose responses to handling and transport stress. North American Journal of Aquaculture, Carbondale, v. 62, n. 1, p. 12-18, 2000.

BITTENCOURT, F. Cultivo de pacu Piaractus mesopotamicus sob diferentes densidades em tanquesrede no reservatório de Itaipu. 2008. 46 f. Dissertação (Mestrado em Zootecnia) - Universidade Estadual do Oeste do Paraná, Toledo, 2008.

CHO, G. K.; HEATH, D. D. Comparison of tricaine methanesulphonate (MS222) and clove oil anesthesia effects on the physiology of juvenile chinook salmon Oncorhynchus tshawytscha (Walbaum). Aquaculture Research, Berlin, v. 31, n. 6, p. 537-546, 2000.
CUNHA, M. A. Anesthesia of silver catfish with eugenol: time of induction, cortisol response and sensory analysis of fillet. Ciência Rural, Santa Maria, v. 40, n. 10, p. 2107 2114, 2010.

DELBON, M. C. E.; PAIVA, M. J. T. R. Eugenol em juvenis de tilápia do Nilo: concentrações e administrações sucessivas. Boletim do Instituto de Pesca, São Paulo, v. 38, n. 1, p. 43-52, 2012.

GOMES, L. C. et al. Efficacy of benzocaine as an anesthetic in juvenile tambaqui Colossoma macropomum. Journal of the World Aquaculture Society, Baton Rouge, v. 32, n. 4, p. 426-431, 2001.

GONÇALVES, A. F. N. et al. Mentol e eugenol como substitutos da benzocaína na indução anestésica de juvenis de pacu. Acta Scientiarum Animal Sciences, Maringá, v. 30, n. 3, p. 339-344, 2008.

GRIFFITHS, S. P. The use of clove oil as an anesthetic and method for sampling intertidal rockpool fishes. Journal of Fish Biology, Oxford, v. 57, n. 6, p. 1453-1464, 2000.

HISANO, H. et al. Tempo de indução e de recuperação de dourados (Salminus brasiliensis), submetidos a diferentes concentrações de óleo de cravo Euginia sp. Acta Scientiarum Biological Sciences, Maringá, v. 30, n. 3, p. 303-307, 2008.

INOUE, L. A. K. A. et al. Clove oil as anesthetic for juveniles of matrinxã Brycon cephalus (Gunther, 1869). Ciência Rural, Santa Maria, v. 33, n. 5, p. 943-947, 2003.

KEENE, J. L. et al. The efficacy of clove oil as an anesthetic for rainbow trout, Oncorhynchus mykiss (Walbaum). Aquaculture Research, Oxford, v. 29, n. 1, p. 89-101, 1998.

OLIVEIRA, J. R. et al. Cloreto de sódio, benzocaína e óleo de cravo-da-Índia na água de transporte de tilápia-do-Nilo. Revista Brasileira de Zootecnia, Viçosa, v. 38, n. 7, p. 1163-1169, 2009.

OSTRENSKY, A. et al. Situação atual da aquicultura brasileira e mundial. In: VALENTI, C. V. et al. (Eds.). Aquicultura no Brasil: bases para um desenvolvimento sustentável. Brasília, DF: CNPq, 2000. p. 354-381.

ROSS, L. G.; ROSS, B. Anesthetic and sedative techniques for aquatic animals. 3. ed. Oxford: Blackwell Science, 2008.

ROUBACH, R. et al. Eugenol as an efficacious anesthetic for tambaqui, Colossoma macropomum (Cuvier). Aquaculture Research, Oxford, v. 36, n. 1, p. 1-6, 2005.

ROUBACH, R.; GOMES, L. Uso de anestésicos durante o manejo de peixes. Panorama da Aquicultura, Rio de Janeiro, v. 11, n. 66, p. 37-40, 2001. 
VIDAL, L.V. O. et al. Utilização do eugenol como anestésico para o manejo de juvenis de pintado (Pseudoplatystoma corruscans). Acta Scientiarum Animal Sciences, Maringá, v. 28 , n. 3 , p. $275-279,2006$.

VIDAL, L. V. O. et al. Influência do peso de juvenis de matrinxã (Brycon cephalus) e tambaqui (Colossoma macropomum) à ação anestésica do eugenol. Revista Brasileira de Saúde e Produção Animal, Salvador, v. 8, n. 3, p. 212-216, 2007.

VIDAL, L. V. O. et al. Eugenol como anestésico para a tilápia-do-Nilo. Pesquisa Agropecuária Brasileira, Brasília, DF, v. 43, n. 8, p. 1069-1074, 2008.
YUKIHIRO, R. G. et al. Diferentes concentrações de benzocaína na indução anestésica do lambari-do-raboamarelo (Astyanax altiparanae). Revista Brasileira de Saúde e Produção Animal, Salvador, v. 9, n. 2, p. 350357, 2008.

WAGNER, G. N. et al. The ability of clove oil and MS222 to minimize handling stress in rainbow trout (Oncorhynchus mykiss Walbaum). Aquaculture research, Oxford, v. 34, n. 13, p. 1139-1146, 2003.

WOODY, C. A. et al. Clove oil as an anesthetic for adult sockeye salmon: field trials. Journal of Fish Biology, Oxford, v. 60, n. 2, p. 340-347, 2002. 